\title{
Benefits of combining neurofeedback and physical therapy for chronic pain: A case study
}

\author{
MarkTrullinger ${ }^{2,3}$, Deepti Pradhan ${ }^{2,3}$, Tom A. Bruns ${ }^{4}$ and Brett B. Clark ${ }^{1,4 *}$ \\ ${ }^{1}$ University of Maryland, School of Medicine, Department of Anatomy and Neurobiology, Baltimore, MD 21230, USA \\ ${ }^{2}$ NeuroThrive, LLC, 1205 York Road, Suite 11, Lutherville, MD 21093, USA \\ ${ }^{3}$ The Chicago School for Professional Psychology, USA \\ ${ }^{4}$ Lifestrength Physical Therapy, Inc., 110 West Road, Suite 105, Towson, MD 21204, USA
}

\begin{abstract}
The combination of neurofeedback and physical therapy is a novel approach for chronic pain treatment. A 66 year old woman with a history of 9 years of chronic head and neck pain, whose progress toward pain reduction had reached a plateau and physical therapy only providing maintenance, was administered 32 sessions of physical therapy and 50 sessions of neurofeedback over a 6 month period. The patient achieved significant reduction in pain, improved sleep, and increase in functional mobility utilizing this combined approach. Pain intensity was reduced from 7.13/10 to $2.30 / 10$, and the number of days with high pain reduced from $4-5$ per week to 1 or less per week. Functionally, the patient increased her sitting tolerance by 2 hours, driving tolerance by 80 minutes and improved average sleep per night by 3 hours. The brain map findings showed reduction in occipital muscle artifact, Alpha peak frequency, and Alpha in the posterior cortex. Overall, this change added up to a $37-42 \%$ improvement in electro-cortical functioning. The importance of these findings is that the combination of physical therapy and neurofeedback may provide an alternative treatment regimen to chronic pain management that lowers the reliance on medication and reduces long-term costs.
\end{abstract}

\section{Introduction}

Chronic spinal pain represents one of the largest medical expenses in industrialized nations. A 2015 analysis of 2012 survey data found that 23 million or $10 \%$ adult Americans reported daily chronic pain [1]. Despite the large financial and functional impacts of chronic pain,; outcomes remain poor. A seven year follow up study found that only $15 \%$ of patients who reported chronic widespread pain at the beginning of the study completely eliminated their symptoms [2]. There is an emerging body of evidence that physical therapy as part of a multidisciplinary care team is an effective treatment strategy for restoring function. Additionally, it has been shown to be effective in reducing pain catastrophizing when applied as part of a multidisciplinary team [3], which is frequently unaddressed in more traditional models of pain management. (NEUROFEEDBACK BACKGROUND) Neurofeedback (NFT), or computer-aided real-time behavioral training of the electroencephalogram (EEG), has been shown to be a clinically effective treatment for some chronic pain for short periods of time conditions in some situations [4] in peer-reviewed journals. Also, posters/presentations at conferences have been presented on NFTB's effectiveness with pain conditions in certain circumstances [511] and fibromyalgia in particular [12]. However, the use of NFT for chronic neck or back pain beyond the immediate effect to relieve pain does not have any published peer-reviewed research. However, there is, with the exception of a hypothesized mechanism by which NFB could directly treat pain [13]. Currently, NFT is through to provide the most benefits through treatment of the co-morbidities such as mental health/psychosomatic factors [14], high blood pressure and cognitive dysfunctions [15], and sleep disorders [8]. And, NFT has been successful at treating the addiction behaviors associated with chronic pain $[9,16]$. It has also been suggested that NFT may also work in pain syndromes by altering brain functioning in a way that changes how brain registers/interprets the pain [14]. In other words, the theory is that NFT alters the how much pain a person "feels" regardless of how many and how much pain signal is sent to the brain. Meaning, the NFT would not directly treat the cause of the pain, but the reaction to it. In fact, NIH recommends the use of Bio- and Neuro-feedback techniques in pain and sleep disorders and gives it the highest level of rating for effectiveness [14,17]; but it was based on primarily EMG biofeedback results and not EEG biofeedback results. However Also, Ibric \& Dragomirescu (2012) also suggest, "It would be erroneous and an oversimplification to suggest that biofeedback/ neurofeedback alone would effectively assist the chronic pain patient to overcome comorbidities associated with their pain." Therefore, combining physical therapy emphasizing manual medicine which has been shown effective for chronic neck pain [18-20] with neurofeedback presents a unique multi-disciplinary care option which may effectively address physical, psychological and neurological aspects of chronic pain.

\section{Methods}

\section{Case description}

The patient is a 66 year old female who was run over by her minivan. The patient's van, a 1997 Dodge Grand Caravan, was parked

Correspondence to: Brett B. Clark, PT, DPT, PhD, University of Maryland, School of Medicine, Department of Anatomy and Neurobiology, Baltimore, MD 21230, USA; Lifestrength Physical Therapy, Inc., 110 West Road, Suite 105, Towson, MD 21204, USA, E-mail: bclark@lifestrengthpt.com

Key words: chronic pain, neurofeedback, physical therapy, QEEG

Received: February 20, 2017; Accepted: March 08, 2017; Published: March 13 2017 
on an incline outside a place of business. Given the patient's short stature, she was unable to release the parking brake with her foot. In order to release the brake she had to open the driver's door, lean to the left, and push the emergency brake with her hand. In attempting to release the brake with her hand, she fell out of the van. The van subsequently rolled backward and the patient's head was run over by the left front wheel. To the patient's good fortune, an off duty EMT witnessed the accident and was able to provide emergency medical care almost immediately. The patient did not lose consciousness at any time during the accident or during transport to the hospital. The patient was transported to R Adams Cowley Shock Trauma Center at the University of Maryland Medical Center via helicopter. The patient sustained fractures of C5 and C6 vertebrae and had severe edema of the left side of the face and head. No skull fractures were identified. Nor was there any neurological trauma identified. The patient underwent surgery to stabilize the fractures at C5 and C6 and fuse those 2 levels via posterior approach. The patient remained in the hospital for 4 days and was discharged home with a neck brace.

The patient reported to the current physical therapist's office 18 months after the initial injury. Prior to her initial evaluation at our office by the current physical therapist, she had undergone 91 physical therapy sessions, 36 acupuncture sessions and 24 massage therapy sessions; but was still suffering with considerable daily, unremitting pain in her head, especially the occipital region and neck. She denied having any radicular symptoms. Past Medical History: Her past medical history was unremarkable for prior neck injury or other health conditions. And, the patient had undergone gall bladder removal in 1995 and hernia repair in 1999. Her medications were Medications: Flexeril and Tylenol PM. Objective Findings: The patient rated her current pain in the head and neck at $7 / 10$, best at $5 / 10$ and worst at 9/10. Her Functional Limitations: Sitting tolerance was limited to $10 \mathrm{~min}$, walking limited to 200 feet and ability to drive significantly impaired due to inability to turn head to see traffic and increased pain sitting in car (8/10 for greater than 10 minutes. The patient exhibited a forward head posture with scapular protraction and thoracic kyphosis. Her cervical range of motion was limited to 30 degrees of forward and backward bending, 25 degrees of right and left side-bending and 45 and 40 degrees of right and left rotation respectively. The upper extremity strength and range of motion was within normal limits. The patient exhibited significant tenderness to palpation of the suboccipital muscles and the occipital region of the head. The patient could only tolerate very light pressure to these areas.

From 18 months post injury to approximately 7 years post injury the patient underwent an average of 45 physical therapy visits per year concentrating on craniosacral techniques and very gentle soft tissue mobilization and neuromobilization techniques. More aggressive treatment and/or exercise created a significant guarding response and increase in pain; therefore, more indirect techniques had to be employed. Over the course of $51 / 2$ years, the patient achieved an increase in cervical range of motion to 50 degrees of forward bending, 45 degrees of backward bending, 40 degrees of left and right sidebending and 70 degrees of left and right rotation. Her sitting tolerance increased from 10 minutes to 2 hours, walking tolerance increased from 200 feet to 1000 feet and patient was able to drive with only minimal restriction versus significant restriction at onset of physical therapy at the 18 month mark with $6 / 10$ pain after 30 minutes. The patient had to wear a TENS unit to go to movies or drive greater than $1 \frac{1}{2}$ hours. Despite increases in cervical range of and improvement in functional limitations, the patient still exhibited significant tightness and tenderness to palpation in the upper trapezius, levator scapulae, sub-occipital muscles, and occipital region of the head. At this juncture ( 7 years post injury, $5 \frac{1}{2}$ years post initiating physical therapy in our office, 315 PT visits later), we began employing a combination of physical therapy and neurofeedback.

\section{Treatment}

At 7 years post injury, $5 \frac{1}{2}$ years post initiating physical therapy in our office and 315 PT visits later, we began employing a combination of physical therapy and neurofeedback. The pain ratings were $5 / 10$ for current pain, $3 / 10$ for best pain, and $7 / 10$ for worst pain. Her pain ratings were $5 / 10$ for current pain, $3 / 10$ for best pain and $7 / 10$ for worst. Functional limitations were walking tolerance of 1000 feet, sitting tolerance of 2 hours and driving limited to 30 minutes with 6/10 pain. And, the patient still required TENS unit for movies and driving greater than $1 \frac{1}{2}$ hours. The cervical range of motion was 50 degrees of forward bending, 45 degrees of backward bending, 40 degrees of left and right side-bending and 70 degrees of left and right rotation. The patient was able to tolerate $50 \%$ more direct palpation and pressure of the levator scapulae, upper trapezius and sub-occipital muscles, but was still limited to 15 minutes of direct palpation and treatment.

A Quantitative Electroencephalogram (QEEG) "brain mapping" was conducted with a Brainmaster Discovery 24e and an Electrocap to record from the 19 points of the 10-20 system to establish a baseline and guide treatment, and it used NeuroGuide and NewMind Databases for the quantitative and normative analyses. The four most significant QEEG findings were an excess muscle artifact that could not be resolved at the sensorimotor and occipital electrodes [21] Figure 1, excess (1-2 clinically relevant deviations above the norm) posterior Alpha [20]. Figure 2 with a higher than the norm peak frequency (1 clinically relevant deviation above the norm) figure 3 , and a global excess of fast or "high" Beta ( 2 or more clinically relevant deviations above the norm).

Physical Therapy sessions were conducted 2 times per week for 45 minutes for 4 weeks with a 4 week break and then 2 times a week for 4 weeks. The 4 week break in treatment was to accommodate insurance visit limits. The treatment consisted of myofascial release techniques and neuromobilization techniques for the first 3 months of combined PT and neurofeedback. The next 3 months of PT treatment involved direct manual therapy techniques for the cervical spine and occiput. These techniques included soft tissue mobilization, joint mobilization and myofascial release.

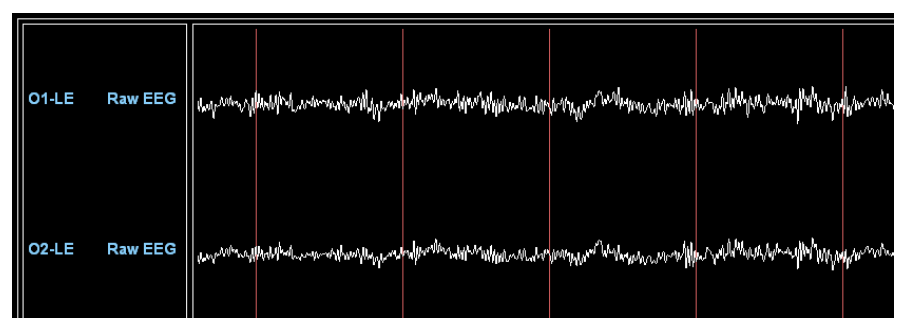

Figure 1. EEG muscle artifact first session.

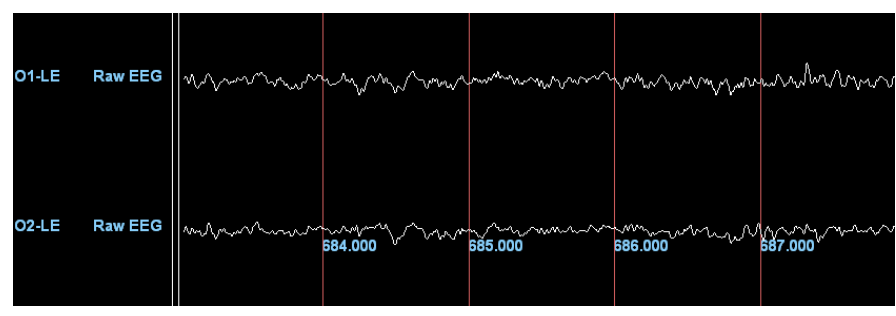

Figure 2. EEG muscle artifact last session. 
orrom quantitative and normative eifour [21] see figure 1 [20]. Figures 2 and 3 Neurofeedback Intervention: The neurofeedback was conducted simultaneously on 3 EEG channels utilizing a Brainmaster Discovery 24e with Avatar software. Channels 1 and 2 were monopolar montages on the sensorimotor strip at $\mathrm{C} 3$ and $\mathrm{C} 4$, respectively. The goals at C3 and C4 were to increase her Sensorimotor Rhythm (SMR) at 12-15 hz, reduce "high" Beta at 20-38 hz, and reduce muscle artifact. Channel 3 was a sequential (bipolar) montage in the occipital cortex at $\mathrm{O} 1-\mathrm{O} 2$. The goals at $\mathrm{O} 1-\mathrm{O} 2$ were to reduce the peak frequency of Alpha to below $12 \mathrm{hz}$, reduce excess Alpha at 9-12hz, reduce "high" Beta at $20-38 \mathrm{hz}$, and reduce muscle artifact. There were 50 neurofeedback training sessions that were 24-30 minutes in length, with, the first half with the eyes open utilizing visual and auditory feedback and the second half with the eyes closed and only auditory feedback. The thresholds adjustments were semi-automatic, with the clinician actively intervening and making alterations throughout the session to the automatic settings and completely turning them off and using manual if necessary to ensure achievement of the best possible learning curve Figure 4. Combined and integrated operant conditioning paradigms were utilized to achieve the maximal learning curve (Table 1).

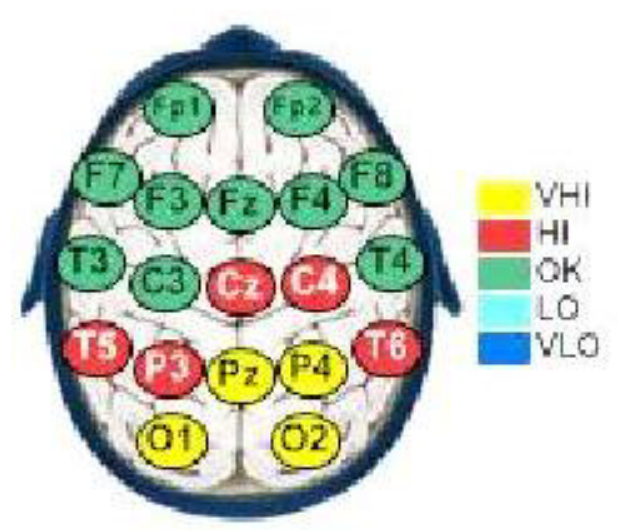

\section{Alpha}

Figure 3. $\mathrm{QEEG}$ eyes closed posterior alpha, pre $(\mathrm{VHI}=$ very high, $\mathrm{HI}=$ high, $\mathrm{OK}=$ good, $\mathrm{LO}=$ low, VLO=very low).

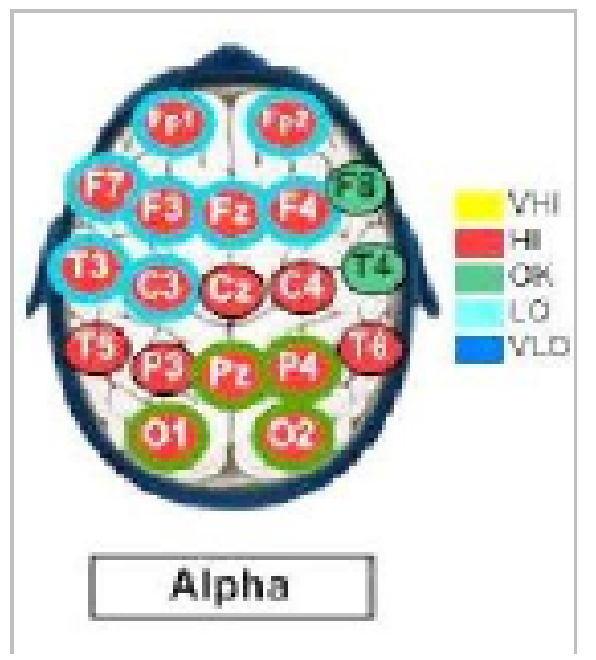

Figure 4. QUEEG eyes closed posterior alpha, post (same convention).
Table 1. Functional change as a result of combining PT and Neurofeedback.

\begin{tabular}{|c|c|c|c|}
\hline & Initiation of PT & $\begin{array}{c}\text { Beginning of PT } \\
\text { and Neurofeedback }\end{array}$ & $\begin{array}{c}\text { After 32 Visits } \\
\text { of PT and 50 } \\
\text { Neurofeedback }\end{array}$ \\
\hline Sitting Tolerance & 1 hour & 2 hours & 3 hours \\
\hline Walking Tolerance & 200 feet & 1000 feet & 2000 feet \\
\hline Driving Tolerance & 10 minutes + & 30 minutes + & 90 minutes* \\
\hline Sleep & 3.5 hours & 3.5 hours & 6.5 hours \\
\hline $\begin{array}{c}\text { Treatment } \\
\text { Tolerance }\end{array}$ & 15 minutes & 15 minutes & 45 minutes \\
\hline Day High Pain & 4 days/wk & 4 days/wk & 1 day/wk \\
\hline Night High Pain & 5 days/wk & 5 days/wk & $<1$ day/wk \\
\hline Pain Intensity & $7 / 10$ & $7.13 / 10$ & $2.30 / 10$ \\
\hline
\end{tabular}

+with TENS unit

*without TENS unit

\section{Results}

Six- months after combining PT and neurofeedback (32 PT sessions), patient was able to go to movies and drive over $1 \frac{1 / 2}{2}$ hours without use of a TENS unit for the first time in 9 years. Additionally, the patient was able to tolerate 45 minutes of direct treatment to the cervical muscles versus 15 minutes at the initiation of neurofeedback. Sitting tolerance increased to over 3 hours and ambulation distance increased to greater than 2000 feet (double the distance before adding neurofeedback). The QEEG analysis showed no visually observable muscle artifact figure 1, a reduction of Alpha peak frequency and dominant frequency to within the norm, a reduction in posterior Alpha to within the norm figure 2, and no significant change in "high" Beta. This change represented just over $20 \%$ regression toward the norm and approximately $50 \%$ of the brain going through reorganization, leading to a total global change of $37-42 \%$ Figure 5 . The pain charting that was initiated with the beginning of the 6 month period neurofeedback intervention showed a decrease in high pain occurrences during the morning from an average of 4 days of occurrences a week to about 1 a week average with a 2 week period of no occurrences of high pain. In fact, all of the high pain times followed a major event that she would have been unable to achieve previously. Likewise, high pain during the evening/night also reduced from an average of 5 days a week of occurrences to less than 1 . The mid-level pain throughout the day remained unchanged, however the amount and type of activities changed and she was able to do significantly more a different activities than she was able to do previously. During occurrences of pain, the overall intensity of the pain reduced from an average of 7.13/10 the first two-weeks to $2.30 / 10$ during the final two weeks. The reductions in both occurrences and the intensity of high and mid-level pain during the evenings also led to a profound effect on her sleep. She went from waking up almost every night because of pain and averaging around 3-4 hours of sleep, to waking up at night an average of 1.5 times a month and getting an average of 6.5 hours of sleep a night (Figures 6, 7 and 8).

\section{Discussion}

The benefits of physical therapy and chronic pain have been shown superior to continued medical care, particularly when emphasizing manual medicine approaches [18]. A 2008 systematic review found mid to high quality evidence to support spinal manipulation and mobilization to produce clinically significant changes in patients [19]. The use of neurofeeedback has been documented as well, though the research is limited. However, the combination of these modalities is a novel approach to chronic pain.

The use of novel approaches in managing chronic pain is particularly important as research continues to reveal the potential 


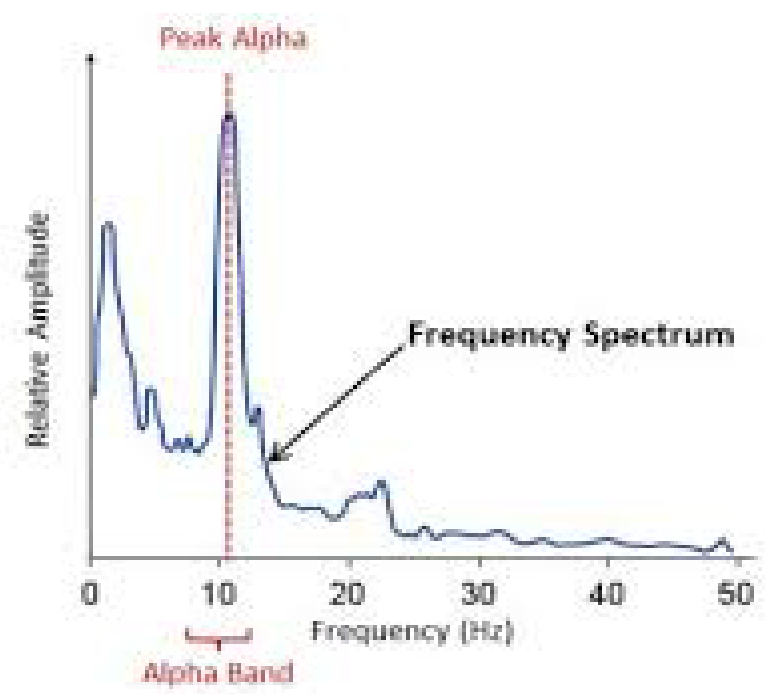

Figure 5. EEG alpha peak frequency.

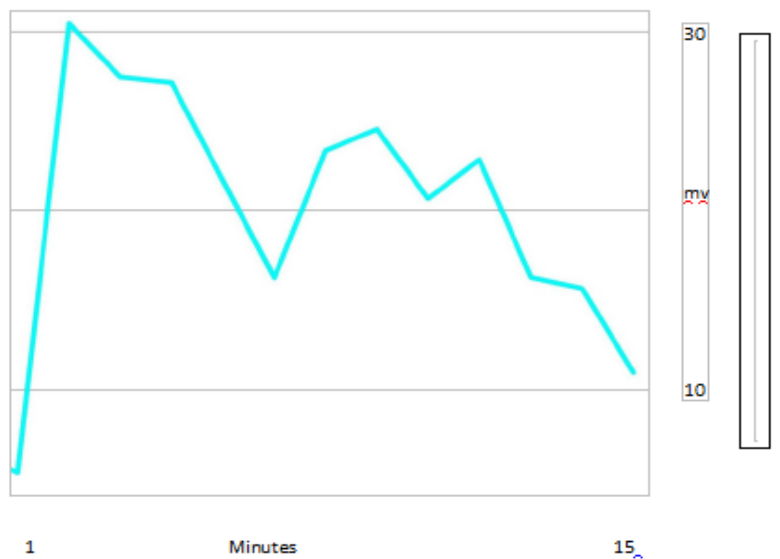

Figure 6. Example of learning curve, decreasing alpha in occipital region.
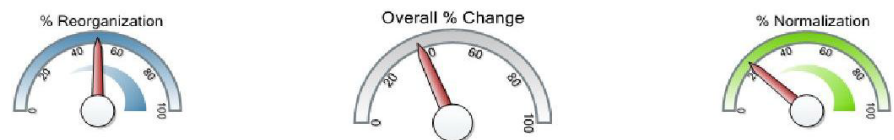

\section{$37 \%$ Overall Change}

Figure 7. Overall change eyes closed.
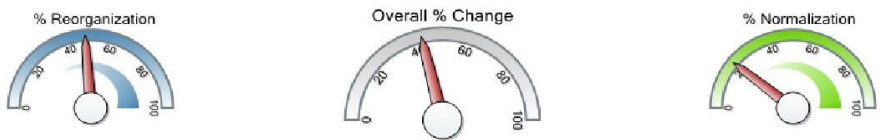

\section{2\% Overall Change}

Figure 8. Overall change eyes open.

dangers of opiate use. A 2015 systematic review found use of opiates increased risk for the following: dependency, increased risk of cardiac events, sexual dysfunction, respiratory complications, and increased risk of motor vehicle accident, but was unable to demonstrate sufficient evidence for improved function or consistently reduced pain [22]. Additionally, recent CDC guidelines illustrate the need to limit the use of opiates as a frontline tool for management of chronic pain, due to these increased risks, and the reduced quality of life and financial burdens associated with them [23].

One of the complicating factors of patients who have developed chronic pain is muscle guarding in the affected areas. This muscle guarding can inhibit the practitioner's ability to assess the tissues effectively and assess joint mechanics. Adding neurofeedback in this case allowed for more direct treatment as muscle guarding and reactivity were reduced after the addition of this modality. This led to significant progress in 32 visits versus a relative plateau in the 315 visits prior to this combination.

Her level of activity during the day increased, including spending all-day at events, riding 6-8 hours in a car, playing sports with her grandchildren, and sitting in seats she was unable to sit in previously. Additionally, reductions in high level pain occurrences and the intensity of the pain during those occurrences was seen despite the increase in activities. Sleep drastically improved due to the reduction in high-level pain occurrences during the night making a full night of pain free sleep the norm instead of the oddity. The use of neurofeedback might be particularly impactful in this area, as new research indicates that cortical arousal, not pre-sleep pain, determines quality of sleep, and neurofeedback is uniquely positioned to alter patterns of cortical excitability [24]. The same paper also concluded that sleep quality was predictive of pain levels and function during the first half of the following day, therefore improved sleep provides a window for improved physical therapy intervention or self- care.

The combination of neurofeedback and physical therapy in this case, a novel approach, demonstrated more significant improvement than physical therapy alone. This may make a case for further research into the combined effects of these modalities and the concurrent use of these modalities in cases of chronic pain. The recommended next step is a larger study of pre-post design in order to investigate if these effects are present across a larger number of chronic pain patients.

\section{References}

1. Nahin RL (2015) Estimates of pain prevalence and severity in adults: United States, 2012. J Pain 16: 769-780. [Crossref]

2. Papageorgiou AC, Silman AJ, Macfarlane GJ (2002) Chronic widespread pain in the population: a seven year follows up study. Ann Rheum Dis 61: 1071-1074. [Crossref]

3. Burns J, Glenn B, Bruehl S (2003) Behavioral Re Cognitive factors influence outcome following multidisciplinary chronic pain treatment: a replication and extension of a cross-lagged panel analysis. Behav Res Ther 163-1182.

4. Jensen MP, Grierson C, Tracy-Smith V, Bacigalupi SC, Othmer S (2007) Neurofeedback treatment for pain associated with complex regional pain syndrome type I. Journal of Neurotherapy 11: 45-53

5. Ibric VL, Jacobs MS (1997) Neurofeedback training in chronic pain associated with post-traumatic stress disorder, and affective disorders. San Diego, CA: [Abstract] Second APEA (ECNS) symposium. May 17.

6. Ibric VL, Kaur S (1999) Neuro-modulation of pain perception through neurofeedback training: long lasting effects on pain control. Poster presented at 9th World Conference on Pain, IASP, Vienna, Austria, Pain, 82, (S272).

7. Ibric VL (2000) Neuro-modulation of pain perception through neurofeedback training long lasting effects on pain control. Proceedings at the 25th AAPM Conference, New Orleans, LA. February 24-26.

8. Ibric VL (2001) EEG biofeedback-neurofeedback training in chronic pain. Poster presented at the International Scientific Conference on Complementary, Alternative and Integrative Medicine Research, San Francisco, CA. May 17-19.

9. Ibric VL (2002) Neurofeedback training enhanced by light and/or electromagnetic closed-loop EEG induces analgesia in patients with neuropathic pain syndromes. Poster presented at the 10th World Conference on Pain, IASP, San Diego, CA, Pain, Suppl., 439-440 (S1338). 
10. Ibric VL (2001) Neurofeedback enhanced by light closed-loop EEG and electromagnetic closedloop EEG in a case of sleep deprivation post methadone withdrawal.

11. Annual Conference of SNR, Monterrey, CA, October 27-30.

12. Kayiran S, Dursun E, Dursun N, Ermutlu N, Karamürsel S (2010) Neurofeedback intervention in fibromyalgia syndrome; a randomized, controlled, rater blind clinical trial. Appl Psychophysiol Biofeedback 35: 293-302. [Crossref]

13. Jensen MP, Hakimian S, Sherlin LH, Fregni F (2008) New insights into neuromodulatory approaches for the treatment of pain. J Pain 9: 193-199. [Crossref]

14. Ibric VL, Dragomirescu LG (2012) Neurofeedback in pain management. Introduction to quantitative EEG and neurofeedback: Advanced theory and applications 2nd ed, 417-451.

15. Ibric VL, Grierson C (1995) Neurofeedback and high blood pressure. Proceedings of 3rd Annual Conference of SSNR, Scottsdale, AZ. April 28-May 1.

16. Guyol G (2006) Neurofeedback: Retraining brain waves healing depression and bipolar disorder without drugs. Part II - The most effective nondrug therapies, pp. $149-157$. NY : Walker and Company. Inc.

17. Lebovits A (2007) Cognitive-behavioral approaches to chronic pain. Primary Psychiatry 9: 48-54.
18. Hoving JL, Koes BW, de Vet HC, van der Windt DA, Assendelft WJ, et al. (2002) Manual therapy, physical therapy, or continued care by a general practitioner for patients with neck pain: a randomized, controlled trial. Ann Intern Med 136: 713. [Crossref]

19. Vernon H, Humphreys BK (2008) Chronic mechanical neck pain in adults treated by manual therapy: a systematic review of change scores in randomized controlled trials of a single session. J Man Manip Ther 16: 42E-52E. [Crossref]

20. Jensen MP, Sherlin LH, Gertz KJ, Braden AL, Kupper AE, et al. (2013) Brain EEG activity correlates of chronic pain in persons with spinal cord injury: clinical implications. Spinal Cord 51: 55-58. [Crossref]

21. Al-Obaidi SM, Nelson RM, Al-Awadhi S, Al-Shuwaie N (2000) The role of anticipation and fear of pain in the persistence of avoidance behavior in patients with chronic low back pain. Spine (Phila Pa 1976) 25: 1126-1131. [Crossref]

22. Chou R, Turner JA, Devine EB, Hansen RN, Sullivan SD, et al. (2015) The effectiveness and risks of long-term opoid therapy for chronic pain: A systematic review for a national institutes of health pathways to prevention workshop. Ann Intern Med 162: 276-286. [Crossref]

23. Have PO. CDC guidelines for prescribing opioids for chronic pain.

24. Tang NK, Goodchild CE, Sanborn AN, Howard J, Salkovskis PM (2012) Deciphering the temporal link between pain and sleep in a heterogeneous chronic pain patient sample: a multilevel daily process study. Sleep 35 : 675-87A. [Crossref]

Copyright: $@ 2017$ Trullinger M. This is an open-access article distributed under the terms of the Creative Commons Attribution License, which permits unrestricted use, distribution, and reproduction in any medium, provided the original author and source are credited. 